\title{
Perioperatives Management bei Patienten mit rheumatoider Arthritis
}

\section{Perioperative Management in Patients with Rheumatoid Arthritis}

Autoren

Institut

\section{U. Hötker, M. Henniger, S. Rehart}

Klinik für Orthopädie und Unfallchirurgie, Markus-Krankenhaus, Akademisches Lehrkrankenhaus der Goethe - Universität, Frankfurt/Main
Schlüsselwörter

- Rheumatoide Arthritis

- perioperativ

- Rheumaorthopädie

Key words

- rheumatoid arthritis

- perioperative

- rheumasurgery
Bibliografie

DOI http://dx.doi.org/

10.1055/s-0042-112358

Online-Publikation: 21.9.2016

Akt Rheumatol 2016; 41:

448-454 @ Georg Thieme

Verlag KG Stuttgart · New York

ISSN 0341-051X

\section{Korrespondenzadresse}

Dr. med. Ulrich Hötker

Klinik für Orthopädie und

Unfallchirurgie

Agaplesion Markus

Krankenhaus

Akademisches Lehr-KH der

Goethe-Universität

60431 Frankfurt

Tel.: +49/69/95332540

Fax: +49/69/9533 2417

ulrich.hoetker@fdk.info

\section{Zusammenfassung \\ $\nabla$}

Die operative Versorgung von Patienten mit rheumatoider Arthritis setzt große Erfahrung voraus, daher sollte die Indikation zur Operation interdisziplinär von einem Rheumaorthopäden in Zusammenarbeit mit seinem internistischen Kollegen getroffen werden. Im Rahmen der Operation müssen bestimmte Strategien verfolgt werden, da die Erkrankung aufgrund ihres systemischen Charakters das perioperative Management durch zahlreichen Komorbiditäten, aber auch durch nötige medikamentöse Therapien und gleichzeitig bestehenden Defektsituationen an mehreren Gelenken, deutlich erschwert. Für die Durchführung der Operation ist daher eine enge Zusammenarbeit zwischen Anästhesie und Chirurgie unerlässlich. Auch die postoperative Nachbehandlung gestaltet sich durch bestehende Defektsituationen an anderen, nicht frisch operierten Gelenken mit ihrer oft stark reduzierten Belastbarkeit schwierig. Zusätzlich können die Erkrankung selbst und ihre medikamentöse Therapie zu Immundepressionen mit schweren Komplikationen führen.

\section{Einleitung}

Bei einer Häufigkeit von 0,65\% der erwachsenen Bevölkerung (ca. 440000 in Deutschland) und Neuerkrankungen von 25 bis 30 bei den Männern und 50 bis 60 bei den Frauen pro 100000 Einwohner handelt es sich bei der rheumatoiden Arthritis (RA) um eine nicht seltene Erkrankung. An der juvenilen idiopathischen Arthritis (JIA) leiden zudem weitere $0,1 \%$ der unter 18 -jährigen (ca. 15000) mit einer Neuerkrankungsrate von ca. 1500 pro Jahr.

Im klinischen operativen Alltag werden sich demnach schon in „normalen“ Einrichtungen immer wieder Patienten mit rheumatoider Arthritis finden, auch wenn sich diese bevorzugt in Zent-

\section{Abstract \\ $\nabla$}

The surgical treatment of patients with rheumatoid arthritis requires a great deal of experience. Therefore, the indication for surgery should be based on interdisciplinary collaboration between a surgeon specialised in rheumatic orthopaedic surgery and an internal medicine specialist. When operating on these patients, specific strategies need to be pursued as the disease significantly complicates the perioperative management due to its systemic nature and numerous comorbidities as well as through drug-related complications and simultaneous defect situations in several joints. Consequently, close collaboration between anaesthesiology and surgery is necessary for the surgical procedure to be carried out successfully. Also the postoperative treatment is complicated by existing defect situations in other joints, often with greatly reduced load bearing capacity. Furthermore, the disease itself and its drug treatment may lead to immunodepression with severe complications.

ren begeben, die sich besonders mit der operativen Versorgung dieser Erkrankung befassen. Aufgrund der klinischen Manifestation, aber auch bei der sehr speziellen Medikation, stellen diese Patienten eine Besonderheit dar.

Die interdisziplinäre Zusammenarbeit von internistischem und orthopädischem Rheumatologen und den Anästhesisten ist hier von großer Bedeutung. Peri- und postoperativ sind bei Bedarf Orthopädietechniker, Physio- und Ergotherapeuten, Sozialarbeiter u.a. eng in die Betreuung mit einzubinden. Die Hinzuziehung eines Psychotherapeuten kann angezeigt sein. Die Betreuung der Betroffenen nach der Entlassung aus stationärer Behandlung muss geregelt und koordiniert sein. 
Das perioperative Management kann grundsätzlich in eine präoperative, eine operative und eine postoperative Phase gegliedert werden.

\section{A Präoperative Phase}

\section{Allgemein}

Schon bei der Planung ist auf die individuelle Situation des Patienten einzugehen (Begleiterkrankungen, Beruf, usw.). Um ein patientengerechtes Vorgehen zu planen, müssen die Lebensumstände bekannt sein: z.B. Anforderungen und Bewältigungsmöglichkeiten. Benötigt der Patient Orthesen oder andere Hilfsmittel? Wird er postoperativ Hilfsmittel benötigen? (Gehstützen, Spezialgehstützen, Orthesen, spezielles Schuhwerk). Oft ist es sinnvoll, die später benötigten Hilfsmittel bereits präoperativ anzuschaffen, um den Gebrauch üben zu können, damit in der postoperativen Situation eine möglichst große Sicherheit in der Nutzung besteht.

Das häusliche Umfeld ist zu eruieren: Besteht eine ausreichende Versorgung durch die Familie, Angehörigen, Hilfsdienste? Werden in der postoperativen häuslichen Phase besondere Bedürfnisse entstehen und wie kann diesen begegnet werden? Die Regelungen hierzu sollten ebenso schon im Vorfeld eingeleitet werden, damit nach Entlassung aus der akut-stationären Behandlung oder aus der Reha-Einrichtung keine Versorgungslücke entsteht.

\section{Indikationsstellung}

Die Operationen bei Patienten mit rheumatoider Arthritis werden aus Gründen der Schmerzlinderung und um Funktionen ganz oder teilweise wiederherzustellen, durchgeführt. Die Entscheidung zur Operation ist in dieser Patientengruppe ganz besonders individuell zu stellen und bei der schwierigen und auch oft nicht ungefährlichen Durchführung der Eingriffe von einem auf diesem Gebiet besonders erfahrenen orthopädischen Rheumatologen zu stellen.

Eine Schwächung des Immunsystems durch die Erkrankung selbst und durch die Medikamente erhöht die Infektanfälligkeit. Die häufigen Zielorgane der Operationen Knochen, Sehne, usw. sind oft in eher „schlechtem“ Zustand, was besondere Vorgehensweisen erfordert.

Oft besteht ein „Operationsstau“ bei den Patienten. Das heißt es finden sich mehrere nötige Operationen. Um ein Vertrauensverhältnis zu dem Patienten aufzubauen ( auch im Hinblick auf später noch evtl. durchzuführende Operationen), ist es sinnvoll, mit einer Operation zu beginnen, die mit ziemlicher Sicherheit ein gutes Resultat erwarten lässt (start with a winner).

Das primär geplante Operationsziel ist immer auch im Zusammenhang mit anderen Insuffizienzen oder Deformitäten in angrenzenden Gelenken zu betrachten. Vielfach ergibt sich die Möglichkeit, durch geschickte Kombination von Operationen an mehreren Gelenken in einer Narkose, funktionale Bewegungsabläufe wieder zu ermöglichen.

Ein weiteres Argument für die Zusammenfassung verschiedener Eingriffe in einer Narkose ist die Tatsache, dass nach oft perioperativ nötigem Absetzen der Rheumamedikation diese bei wieder Ansetzten postoperativ in einem Teil der Fälle nicht mehr wirkt. Sinnvoll ist es, mit Operationen an der unteren Extremität zu beginnen, um eine Gehfähigkeit des Patienten zu erhalten, die für die Teilnahme am täglichen Leben von ausschlagender Wichtigkeit ist.
Die Erstellung eines sinnvollen Strategie-Planes für die Abfolge der Operationen sollte in enger Absprache mit dem Patienten erfolgen.

Neben diesen sinnvollen Planungen ist jedoch zu beachten, dass Zustände mit Nervenkompressionen oder gar Infekten in der Dringlichkeit an erster Stelle stehen.

Für jede Operation bedarf es einer Abstimmung mit den anderen Behandlern (z.B. der internistischen Rheumatologie) für die Eingriffe selbst und für die entsprechende zeitliche Terminierung [1].

\section{Aufklärung}

Als systemische Erkrankung sind meist nicht nur die Gelenke von der Erkrankung betroffen, sondern alle Gewebe und innere Organe können einbezogen sein. Aufgrund der oft reduzierten Mobilisation, aber auch wegen des oft vorgeschrittenen (meist postmenopausalen) Alters und einer häufigen langzeitigen Kortisontherapie besteht praktisch regelhaft eine begleitende Osteoporose.

Die sich aus dem individuellen „Betroffen-Sein“ des Patienten an diesen Krankheits-Manifestationen ergebenden persönlichen Risiken müssen in der Aufklärung mit dem Patienten intensiv erörtert werden und das geplante sinnvolle Vorgehen einvernehmlich abgesprochen werden. Auch nicht direkt operationsbedingte Risiken sind hierbei anzusprechen.

Durch operative Interventionen lässt sich oft kein „Normalzustand“ erreichen, mögliche Reduktionen von Einschränkungen (als „Teilerfolg“) sind dem Patienten als erlebte Verbesserungen zu erklären.

Mit dem Patienten ist auch zu besprechen, dass Stabilität schaffende Operationen (z.B. Arthrodesen), trotz gewisser Funktionsverminderung die Gebrauchsfähigkeit der Gliedmaße ganz erheblich steigern können.

Der postoperative Heilverlauf kann durch die benötigten, oft immunsupprimierenden medikamentösen Therapien kompliziert werden, mit den hieraus ableitbaren chirurgischen und auch infektiösen Problemen. Auch diese Inhalte müssen verständlich erörtert werden.

Chirurgische Interventionen bei dieser Patientengruppe mit einer überdurchschnittlich hohen Eingriffsfrequenz sollten deshalb sinnvollerweise nur mit der entsprechend dringenden Indikation durchgeführt werden, wenn der Verlauf der RA die Intervention erzwingt oder der Verlust wichtiger Gelenkfunktionen droht, bzw. wichtige Gelenkfunktionen für die tägliche Aktivität der Patienten wieder hergestellt werden müssen. Immer ist auch über operative wie konservative alternative Vorgehensweisen aufzuklären.

Die besonderen Affektionen des muskulo-skelettalen Apparates mit den Manifestationen der RA an Knochen, Gelenken, Sehnen, Schleimhäuten, Bändern und Muskeln werden im Allgemeinen vom orthopädischen Rheumatologen behandelt.

\section{Abklärung durch den internistischen Rheumatologen}

Der systemische Charakter der RA ermöglicht die Beteiligung nahezu aller Organe am Krankheitsgeschehen. Häufig befallen von der Erkrankung sind Herz, Lunge, Nieren, Augen und Leber. Mehr als 2/3 der Patienten weisen mindestens eine Ko-Morbidität auf. Zu den genannten Organ-Affektionen kommen häufig noch Erkrankungen wie Diabetes mellitus, Hypercholesterinämie, Adipositas und Osteoporose. Das Risiko in dieser Patientengruppe, an Herz-Kreislauferkrankungen zu sterben, ist ca. 5-fach höher als in der Normalbevölkerung. Insgesamt ist die Lebens- 
erwartung der Patienten mit RA um durchschnittlich 10 Jahre verkürzt im Vergleich zur Normalbevölkerung. Dies gilt vor allem bei nicht oder medikamentös schlecht eingestellten Patienten.

Bei den potentiell hohen Risiken bei Operationen ist es besonders wichtig, Patienten bei nicht vermeidbaren Eingriffen die in einen möglichst guten Zustand zu bringen. Eine Vorstellung beim internistischen Rheumatologen ist meist unabdingbar. Diese Vorstellung und eine Therapie zur Optimierung der Organfunktionen sollte bereits lange Wochen vor dem vereinbarten (elektiven) OP-Termin erfolgen, um genügend Zeit für eine Besserung und medikamentöse Einstellung zur Verfügung zu haben. Immer sollten eine Hypertonie, Herzinsuffizienz, Herzrhythmusstörungen, eine koronare Herzerkrankung, aber auch Leber und Nierenfunktion medikamentös optimal eingestellt werden.

Die zu operierenden Patienten sind häufig fortgeschrittenen Alters. Hierbei kommen zusätzlich noch die altersspezifischen Erkrankungen hinzu. Oft handelt es sich hierbei um Kachexie, Malnutrition, Durchgangssyndrom im Rahmen der stationären Therapie sowie eine Demenz. Unter diesen Gesichtspunkten ist es ratsam, ggf. auch einen Geriater hinzu zu ziehen.

Als weiteres abzuwägendes Risiko ist die der Grunderkrankung geschuldete Immuninsuffizienz (krankheitsbedingt oder auch durch medikamentöse Therapie) zu bedenken.

\section{RA spezifische Medikation}

Heute stehen zur Behandlung der rheumatoiden Arthritis potente Medikamente zur Verfügung. Die Behandlung wird von Anfang an sehr aggressiv geführt („hit hard and early“), um eine frühe Remission und evtl. sogar eine Ausheilung der Erkrankung zu erreichen. Die meisten Patienten sind mit einem DMARD/Immunsuppressivum und einem Biologikum eingestellt. Es handelt sich um immunsupressive und immunmodulierende Medikamente, die dadurch auch Einfluss auf die Infektabwehr und die Wundheilung haben. Die Erkrankung ist durch diese Therapie oft auf niedrigem Krankheitsniveau oder auch weitgehend symptomfrei eingestellt. Für Operationen erhöht sich aber das Risiko für Infekte und/oder Wundheilungsstörungen. Das Absetzen der Medikation erniedrigt zwar die OP-Risiken, jedoch ist nicht sicher, ob nach einem Medikamenten-Absetzen für die Operation und die postoperative Phase bei erneutem Ansetzen der Therapie mit diesen Pharmaka auch wieder der gleiche gute Effekt, wie präoperativ beobachtet, einsetzt. Das heißt, es ist nicht sicher zu gewährleisten, dass das Medikament nach Wiederaufnahme der Therapie noch oder noch so gut wirkt.

Die Therapie mit Kortison wird präoperativ soweit wie möglich in der Dosis reduziert. Wegen einer möglichen Nebenniereninsuffizienz bei Langzeittherapie von mehr als $10 \mathrm{mg} / \mathrm{d}$ erhöhen wir die Dosis perioperativ für ca. 3 Tage („Stoß“), um der Gefahr einer Addison-Krise zu begegnen.

Beobachtungen im klinischen operativen Alltag haben ein Handlungsschema entstehen lassen, das in unserer Klinik praktiziert wird und in $\odot$ Tab. 1 vorgestellt wird.

\section{Methotrexat}

Methotrexat ist bezüglich der perioperativen Gabe das bestuntersuchte DMARD. Studien von Grennan et al. [2] und Sany et al. [3] zeigten randomisiert und prospektiv mit fortgesetzter oder unterbrochener perioperativer Gabe von MTX, dass in der Gruppe mit kontinuierlicher Gabe deutlich weniger Komplikationen auftraten. Es zeigte sich kein Anstieg der Infektionsrate. Bezüg-

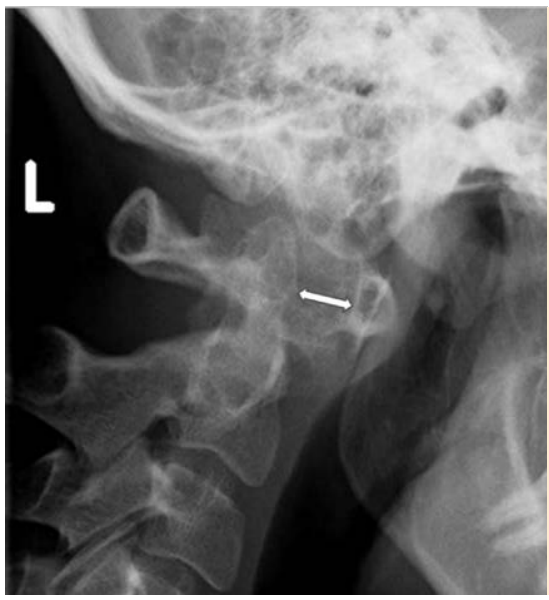

Abb. 1 Seitliche HWS mit atlanto-axialer Insuffizienz.

Tab. 1 Mögliches perioperatives Management von Rheuma-Medikationen.

\begin{tabular}{|c|c|}
\hline \multicolumn{2}{|l|}{ DMARDs } \\
\hline Methotrexat & $\begin{array}{l}\text { kein Absetzen (bei hoher Dosis evtl, } \\
\text { Reduktion) }\end{array}$ \\
\hline Sulfasalazin & kein Absetzen \\
\hline Hydroxychloroquin & kein Absetzen \\
\hline Leflunomid & $\begin{array}{l}\text { Infektrisiko gering: kein Absetzen, sonst: } \\
5 \text { Tage vor dem Eingriff Auswaschen mit } \\
\text { Cholestyramin }\end{array}$ \\
\hline \multicolumn{2}{|l|}{ Immunsupressiva } \\
\hline Azathioprin & 1-2 Tage präop pausieren \\
\hline Cyclosporin & 1-2 Tage präop pausieren \\
\hline Mycophenolat-Mofetil & 1-2 Tage präop pausieren \\
\hline Cyclophosphamid & 4-6 Wo präop pausieren \\
\hline \multicolumn{2}{|l|}{ Biologika } \\
\hline Etanercept & 2 Wo präop pausieren \\
\hline Adalimumab & 4 Wo präop pausieren \\
\hline Infliximab & 3-4 Wo präop pausieren \\
\hline Golimumab & 4 Wo präop pausieren \\
\hline Certolizumab & 4 Wo präop pausieren \\
\hline Rituximab & mind. 2 Monate präop pausieren \\
\hline Abatacept & 4 Wo präop pausieren \\
\hline Tocilizumab & 4 Wo präop pausieren \\
\hline
\end{tabular}

lich der Wundheilungsstörungsrate ergaben sich keine Unterschiede.

Bei einer Dosierung bis $20 \mathrm{mg} /$ Woche kann die kontinuierliche perioperative Gabe von Methotrexat empfohlen werden [4]. Zu gleichen Empfehlungen kommen Visser et al. [5] aufgrund einer Literaturrecherche der multinationalen 3E-Initiative.

\section{Leflunomid}

Tanaka et al. [6] sowie Fürst et al. [7] berichten über eine Studien mit kontinuierlicher Gabe und unterbrochener Gabe von Leflunomid. Bezüglich der Infektionsrate ergaben sich hier keine Unterschiede. Die Studie mit kontinuierlicher Leflunomid-Gabe hat jedoch im Vergleich zu kontinuierlicher Methotrexat Gabe ein 40,6\% höheres Risiko für Wundheilungsstörungen.

Bei kleinen Eingriffen und zugleich niedrigem Infektionsrisiko kann die kontinuierliche Medikation empfohlen werden. Bei größeren Eingriffen sollte die Substanz 5 Tage präoperativ durch Cholestyramin ausgewaschen werden. 


\section{Azathioprin, Sulfasalazin}

Für Azathioprin berichten Barnard et al. [8] bei handchirurgischen Patienten über keinen Zusammenhang mit perioperativen Infektionen. Escalante und Beardmore [9] fanden jedoch in einer retrospektiven Studie eine Verdopplung der Komplikationsraten.

Bei kurzer Halbwertszeit der Substanz sollte sie 1-2 Tage präoperativ pausiert werden. Bei so kleinen Zeiträumen ist noch nicht mit einem Aufflammen der rheumatoiden Arthritis zu rechnen.

Für Sulfasalazin haben Den Broeder et al. [10] ein im Vergleich zu anderen Risikofaktoren erniedrigtes Risiko gefunden. Sulfasalzin hat nur eine geringe immunsuppressive Wirkung und kurze Halbwertszeit von 4 bis 5 Stunden, sodass es perioperativ weitergeführt werden kann.

\section{Hydroxychloroquin, Chloroquin}

Bei einer langen Halbwertszeit von 40 bis 50 Tagen und nur geringer immunsuppressiver Wirkung empfiehlt es sich, die Substanzen kontinuierlich zu geben.

\section{Anti-TNF-a-Therapie}

Bongartz et al. [11] berichten über eine deutlich erhöhte Rate an Wundinfektionen bei Anti-TNF-a-Therapie.

Den Broeder et al. [10] sowie Kawakami et al. [12] fanden in Studien bei TNF-a-Medikation ein erhöhtes Infekt- und Wundheilungsrisiko. Gilson et al. [13] fanden bei hüftprothetischen Eingriffen eine erhöhte Infektrate unter Anti-TNF-a-Therapie.

Die Deutsche Gesellschaft für Rheumatologie empfiehlt eine Pausierung der Medikation zumindest bei größeren Eingriffen für 2 Halbwertszeiten. Es ergeben sich dann Pausenzeiten für Etanercept von 9 Tagen, für Infliximab von 16-20 Tagen, für Golimumab von 24 Tagen, für Adalimumab und Certolizimumab von 28 Tagen [4]. Bei kleinen Eingriffen mit nur sehr geringem Risiko auf Infektionen und andere Komplikationen können Biologika auch perioperativ gegeben werden.

\section{Tocilizumab (Antikörper gegen Interleukin-6-Rezeptor)}

Hirao et al. [14] berichten in einer retrospektiven Studie von 161 Operationen, dass in 20 Fällen die Wundheilung verzögert und in 3 Fällen eine Infektion zu verzeichnen war.

\section{Rituximab (CD 20 Antikörper)}

Toussirot et al. [15] berichten aus einer laufenden Kohortenstudie über 95 orthopädische Operationen, bei denen postoperative Komplikationen in 7,4\% gefunden wurden (Infektion, 1-mal Tod in Sepsis)

\section{Abatacept (Inhibitor von CD-28 abhängigen T-Zell Antworten/„T-Zell-Co-Stimulationsblocker“)}

$\mathrm{Zu}$ Abatacept gibt es wenige Daten. Nishida et al. [16] berichten über eine Fallserie von 7 Patienten. Sie kommen zu dem Schluss dieses Medikament 2-3 Wochen vor dem Eingriff abzusetzen. Eine Leitlinie zur Biologikatherapie perioperativ gibt es zur Zeit noch nicht. Es handelt sich hier um Expertenempfehlungen.

\section{Anästhesie}

Der Anästhesie kommt in der operativen Versorgung von Rheumapatienten eine wichtige Rolle zu. Die Patienten sind oft multimorbid. Aufgrund der Organbeteiligungen am systemischen Krankheitsgeschehen können wichtige Funktionen deut- lich eingeschränkt sein, die die Narkoseführung stark beeinflussen können. Die Patienten sollten dem Anästhesisten möglichst frühzeitig präoperativ vorgestellt werden. Es muss genügend Zeit verbleiben, eventuell nötige präoperative Konsultationen anderer Fachgebiete noch bis zu einem geplanten OP-Termin einholen zu können. Die Entscheidung über die Art des Narkoseverfahrens hängt von der Art und Dauer des Eingriffes sowie auch von Besonderheiten der Erkrankung im individuellen Verlauf und von den Wünschen des Patienten ab.

Insbesondere bei langjährigen Verläufen der rheumatoiden $\mathrm{Ar}$ thritis sind oft Gelenke im Bereich des Kopfes vom Krankheitsgeschehen miterfasst. Hier sind insbesondere die Kiefergelenke, der Kehlkopf und die oberen Halswirbelgelenke zu nennen. Bei Beteiligung der Kiefergelenke findet sich oft eine verminderte Öffnungsfähigkeit des Mundes, die zu Schwierigkeiten bei der Intubation führen kann. Hier ist ggf. eine fiberoptisch kontrollierte Intubation nötig. Wenn die Cricoarytenoidgelenke des Kehlkopfes einbezogen sind, kann dieses zu einer deutlichen Verengung im subglottischen Bereich führen, die auch die Intubation erheblich erschweren kann. In diesem Fall muss ebenfalls eine fiberoptisch kontrollierte nasotracheale Intubation erfolgen.

Die Halswirbelsäule ist bei langjährigen Erkrankungsverläufen bis zu $80 \%$ in die Pathologie mit einbezogen. Hiervon ist wiederum die proximale HWS zu 70\% betroffen. Die häufigste Manifestation ist die atlanto-axiale Subluxation [17] als Folge der erosiven Synovialitis und der folgenden Zerstörung der atlanto-axialen und atlanto-odontoidalen Gelenke, sowie der Bursa zwischen Dens und Ligamentum transversum atlantis. Hierdurch entsteht eine Instabilität, die im Verlauf zu einer Kompression der Medulla oblongata führen kann. Pannusbildungen können zu einer weiteren Kompression führen. Insbesondere bei Beugebewegungen kann der Dens bei Zerstörung des Ligamentum transversum (fehlende dorsale Bewegungssperre) die Medulla oblongata schädigen (8-fach erhöhtes Risiko).

In unserem Patientengut mit rheumatoider Arthritis wird prinzipiell präoperativ eine seitliche Aufnahme der HWS mit Zentrierung auf C1 samt Darstellung des harten Gaumens angefertigt. Bei Auffälligkeiten werden weitere Bilder in Flexion und Extension veranlasst. Evtl. müssen die Untersuchungen um CT und MRT ergänzt werden.

In den seitlichen Aufnahmen wird das anteriore atlanto-dentale Intervall (AADI, der Abstand zwischen ventralem Dens und Hinterrand des vorderen Atlasbogens) gemessen. In Flexionsaufnahmen darf das Intervall $3 \mathrm{~mm}$ betragen, ab $6 \mathrm{~mm}$ und mehr ist von einer Insuffizienz auszugehen.

Weiterhin kann das posteriore atlanto-dentale Intervall PADI (Abstand hintere Densbegrenzung zum Vorderrand des hinteren Atlasbogens) gemessen werden. Hierbei wird der verbliebene Raum für das hindurchziehende Rückenmark beurteilt. Bei einem Intervall kleiner als $14 \mathrm{~mm}$ ist mit einer deutlichen Insuffizienz und neurologischen Symptomen zu rechnen (Myelomalazie).

Bei derartigen Befunden im Rahmen einer präoperativen Diagnostik ist höchste Vorsicht geboten. Der Patient sollte dann ggf. einem (orthopädisch-rheumatologischen) Wirbelsäulenchirurgen vorgestellt werden. Häufig muss dann noch vor der eigentlich geplanten Operation die Stabilisierung der Halswirbelsäule erfolgen. Möglichkeiten hierzu bieten sich z.B. in einer transartikulären Verschraubung zwischen Dens und Axis mit einem Knochenspan zwischen den Wirbelbögen 1 und 2. Eine weitere Möglichkeit ist eine Pedikelverschraubung HWK 1 und 2. Es 
kommt hierdurch zu einer Aufhebung der Beweglichkeit zwischen HWK 1 und 2, was auch die gefährliche („sudden death“) atlanto-axilale Insuffizienz beseitigt.

Weiterhin kann in den seitlichen Röntgenaufnahmen der HWS die McGregor-Linie beurteilt werden. Sie wird von der Hinterkante des harten Gaumens zum tiefsten Punkt des Occiputs gezogen. Liegt die Spitze des Dens mehr als $4,5 \mathrm{~mm}$ oberhalb dieser Linie, kann von einer basilären Impression ausgegangen werden. Auch hier kann eine Schädigung der Medulla oblongata vorliegen und es sollte in diesen Fällen eine chirurgische Stabilisierung vorgenommen werden.

Intubationsnarkosen sind für Patienten mit rheumatoider Arthritis mit deutlich mehr Risiken behaftet als für die gesunde Bevölkerung. Unsere Empfehlung lautet daher, soweit möglich, lokale und regionale Anästhesieverfahren zu verwenden $(\bullet$ Abb. 1).

\section{B Operative Phase}

$\nabla$

\section{Operationstaktik}

Aufgrund der multilokulären Erscheinungsform und des oft steten Fortschreitens der Erkrankung müssen viele Patienten mit rheumatoider Arthritis immer wieder operiert werden. Ziel der Eingriffe ist es, Schmerzen zu lindern und Funktionen ganz oder teilweise wieder herzustellen. Es resultiert für viele Patienten daraus eine hohe Operationsfrequenz. Um die Zahl der Eingriffe mit Krankenhausaufenthalt gering zu halten, ist es sinnvoll, wenn immer möglich, mehrere Operationen in einer Sitzung und einer Narkose zusammenzufassen. Für den Patienten reduziert sich dadurch die Anzahl der Krankenhausaufenthalte.

Wichtiges Ziel in der operativen Vorgehensweise ist es, den Patienten beweglich (gehfähig) zu erhalten, um eine möglichst hohe Teilnahmefähigkeit an Aktivitäten des täglichen Lebens zu erhalten. Es kommt daher den die Gehfähigkeit erhaltenden oder wiederherstellenden Eingriffen an den unteren Extremitäten Vorrang zu.

Ein weiteres bewährtes Vorgehen ist, zunächst die stammnahen Gelenk zu sanieren und dann zu den körperferneren Gelenken fortzuschreiten. Sinnvoll ist, die körpernahen Gelenke möglichst beweglich zu erhalten, weiter peripher steht oft eher die Stabilität im Vordergrund.

\section{Lagerung, Temperaturregulation}

Bei Patienten mit rheumatoider Arthritis ist noch mehr als bei allen anderen Patienten größte Sorgfalt bei der Lagerung zur Operation zu gewährleisten. Die Operationstische sind zwar alle mit Gelauflagen gut gepolstert, werden jedoch von den Patienten fast ausnahmslos als „hart“ beurteilt. Die Liegezeit auf den Tischen ist daher kurz zu halten. Unnötige Verlängerungen - z. B. durch zu frühes in-den-OP-bestellen der Patienten - um Wechselzeiten zu optimieren, ist für die Patienten nicht von Vorteil.

Bei der Lagerung auf den Tischen sind Besonderheiten der Rheumapatienten zu beachten. Bei vielen Patienten bestehen Kontrakturen in Gelenken. Diese Extremitäten und oft auch der deformierte Rücken sind entsprechend mit zusätzlichen Gelkissen und Tüchern so abzupolstern, dass kein Zug auf die kontrakten Gelenke kommt. Die oft auch sehr kachektischen Patienten müssen vor allem an den Auflageflächen zusätzlich weich gebettet werden.
Bei der Auslagerung von Extremitäten ist der Freiheitsgrad der Gelenke in der Beweglichkeit zu beachten. Eine Extremität darf nicht längerzeitig im maximalen Endbewegungspunkt des entsprechenden Gelenkes gelagert werden. Die Lagerung muss insgesamt komfortabel für den Patienten sein, damit er, insbesondere wenn regionale Anästhesieformen zur Anwendung kommen, bei längeren Eingriffen, bei dabei nicht veränderbarer Lage, keine Schmerzen leidet.

Die Haut der Patienten ist oft besonders problematisch. Durch vielfach jahrelange Therapie mit Kortikosteroiden hat sich bei einer Vielzahl der Patienten die typische „Kortisonhaut“ entwickelt, mit hochgradiger Atrophie und ebenso hochgradiger Vulnerabilität. Es empfiehlt sich hier, die Patienten oft im wahrsten Sinn des Wortes „in Watte zu packen“. Besonders problematisch sind in diesen Fällen die Kleberänder der chirurgischen Abdecktücher. Beim Entfernen der Tücher nach der Operation können oberflächliche Hautschichten an ihnen hängen bleiben, was zu großflächigen Wunden der Patienten führen kann. Es ist zu empfehlen, die Extremitäten, auch wenn peripher operiert wird, möglichst weit proximal abzudecken, da in diesem Bereich die Haut oft in etwas besserem Zustand ist.

Das Anlegen der Blutsperre, die für viele Operationen gern genutzt wird, ist auf ein Minimum der Eingriffe zu beschränken. Durch den Druck der Manschetten können bei der oft atrophischen Extremität und den schlechten Hautverhältnissen Druckschäden entstehen.

Patienten empfinden die Temperaturen im OP meist als kalt. Im OP Saal sollten Temperaturen zwischen 22 bis $26+{ }^{\circ} \mathrm{C}$ konstant bestehen. Die gemessene Körpertemperatur des Patienten sollte auf dem normalen Niveau von $37^{\circ} \mathrm{C}$ gehalten werden. Auskühlung der Patienten führt zu mehr infektiösen Komplikationen und zu Verschlechterung der Blutgerinnung. Zur Wärmung der Patienten sind regelhaft Wärmematten unter und auf dem Körper zu verwenden. Alle nicht zur Operation oder zur Narkoseführung benötigten Bereiche des Körpers sind zusätzlich mit Decken einzuhüllen. Das Risiko ungewollter intraoperativer Hypothermie steigt auf Patientenseite durch Zugehörigkeit zu einer ASA Klasse höher 1, niedriges Körpergewicht, Diabetes mellitus und diabetische Neuropathie. Anästhesiologische Gefährdungsfaktoren sind: Allgemeinanästhesie, lange Anästhesiedauer, Höhe der spinalen Blockade bei rückenmarksnahen Anästhesien und Menge sowie Temperatur von Infusionslösungen und Bluttransfusionen. Auf chirurgischer Seite sind die Größe des chirurgischen Eingriffs, eine Operationsdauer über $2 \mathrm{~h}$ und hohe Volumina an Spülflüssigkeiten von Relevanz (S3 Leitlinie [18]).

2007 zeigten Romlin et al. [19] sowie Rajagopalan et al. [20] einen erhöhten Bluttransfusionsbedarf und eine verlängerte Blutung bei Hypothermie. Frank et al. [21] konnten zeigen, dass die Aufrechterhaltung der normalen Körpertemperatur die Risiken für kardiale Problematiken reduziert. Forbes et al. [22] untersuchten zahlreiche Studien zu Wundinfektionen und Wundheilungsstörungen. Bei Hypothermie fand sich jeweils eine gesteigerte Rate an diesen Komplikationen.

\section{Operation}

Bereits mit dem Hautschnitt können Komplikationen verhindert werden. Ein minimal invasiver Zugang kann bei normalem Zustand der zu inzidierenden Haut schonend und angezeigt sein. Bei Patienten mit RA sind die Gewebe jedoch durch die Erkrankung und evtl. auch durch schon früher erfolgte Operationen 
geschädigt. Eine kleine Inzision würde hier zu einer Dehnung der Gewebe führen, um den benötigten Überblick über die Strukturen zu erhalten. Diese Dehnungen führen zu Wundheilungsstörungen, insbesondere der Hautränder. Unserer Erfahrung nach ist auf einen ausreichend großen Schnitt zu achten, damit keine Spannungen in den Weichteilen auftreten, die sonst sehr leicht zu Wundrandnekrosen und anschließend drohendem Infekt führen. Bei der Präparation in die Tiefe der Wunde müssen oberflächlicher liegende Strukturen mit Wundhaken beiseite gehalten werden. Auch hierbei ist ein ausreichend großer Hautschnitt eine Erleichterung. Jedes forcierte Ziehen an den Haken kann sich bei Rheumapatienten in Wundnekrosen und Schädigungen der Gewebe später bemerkbar machen. Der abschließende Verband ist ohne Druck aufzulegen. Bei „Kortisonhaut“ muss auf Klebeverbände verzichtet werden. Wundgazeauflagen verhindern weitgehend das Festkleben von Verbandmaterial mit der Haut und der Wunde. Beim Anlegen von Orthesen oder Gipsverbänden ist bei diesen Patienten auf eine extrem gute Polsterung zu achten. Günstig ist es, wenn bereits präoperativ Schienen und Hilfsmittel angepasst wurden. Regelungen betreffend Bluttransfusionen intraoperativ sind generell wie bei allen anderen Patientengruppen vorzunehmen. Transfundiert wird bei Hb-Werten $\leq 7 \mathrm{~g} / \mathrm{dl}$. Ausnahmen bilden alle Fälle, bei denen aufgrund von Nebenerkrankungen die Transfusion auch bei höheren Hb-Werten angezeigt ist. Patienten mit rheumatoider Arthritis bilden aufgrund ihrer Ko-Morbiditäten hier oft eine Ausnahme (z. B. kardiale Erkrankungen). Eine „single-shot“-Antbiotikumprophlylaxe bei größeren Operationen wird regelmäßig gegeben. Bei Rheumapatienten sollte aufgrund ihrer Immunsuppression (krankheitsbedingt, medikamentös) mit der Prophylaxe großzügig verfahren werden.

Rheumapatienten tragen ein hohes Operationsrisiko. Hierzu zählt zunächst das dem Eingriff inhärente Risiko. Hinzu kommt dasjenige der rheumatischen Erkrankung selbst. Die Abwehrlage der Patienten ist moduliert durch die Krankheitsaktivität. Weitere Faktoren sind die Dauer der Erkrankung und die resultierenden Veränderungen. Durch die dauerhafte Erhöhung von Entzündungsmediatoren mit hohen Spiegeln von zirkulierenden Immunkomplexen werden katabole Mechanismen gefördert, die mit einer erhöhten Rate an Wundheilungsstörungen und Infekten einhergehen. Hinzu kommen für „Rheumatiker“ typischen Ko-Morbiditäten, die das Risiko weiter erhöhen. Zu nennen sind hier chronische Lungenerkrankungen, kardiale und renale Insuffizienzen, Diabetes mellitus und zusätzlich besonders Nikotin- und Alkoholkonsum. Bereits einliegende Gelenkendoprothesen, durchgemachte Gelenk- und Weichteilinfektionen und schwere Gelenkzerstörungen erhöhen das Risiko für infektiöse Komplikationen weiter. Im Vergleich zu Arthrosepatienten haben Rheumapatienten per se ein deutlich erhöhtes Risiko [23].

\section{Postoperative Phase}

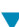

\section{Schmerztherapie}

Bei Nutzung von Nervenblockaden schon in der Operationsphase hält die Wirkung und damit auch die Schmerzfreiheit evtl. noch Stunden an. Verbunden mit den Blockaden ist jedoch auch eine mögliche motorische Beeinträchtigung. Bei Operationen an der unteren Extremität sind die Patienten damit evtl. noch am Tage nach der Operation nicht sicher mobilisierbar (Gefahr von
Stürzen). Dieses hindert auch die frühe krankengymnastische Therapie.

In der frühen postoperativen Phase kommt wie bei allen Operationen ein Stufenschema der Schmerztherapie zur Anwendung. Eine Möglichkeit des Vorgehens sei kurz vorgestellt. Bei kleinen Eingriffen sind die Mittel der Wahl Metamizol, verschiedene NSAR, so dazu zugelassen, evtl. Tramadol o.a. Bei größeren Eingriffen wird das Spektrum um Oxycodon und Tilidin erweitert. Bei großen Operationen mit starken Schmerzen kommen Piritramid und evtl. ein PDK oder eine PCA hinzu.

\section{Physiotherapie, Mobilisation}

Die frühe Mobilisation postoperativ ist von großer Wichtigkeit. Sie kann kompliziert werden durch Komorbiditäten. Aufgrund der gleichzeitigen Ausprägung der Erkrankung an verschiedenen Gelenken bei Rheumapatienten können weitere Problematiken entstehen. So ist es vielen Patienten aufgrund des „Befalls“ beider unteren Extremitäten nicht möglich, ein Bein zu entlasten oder auch nur teilzubelasten. Daher sollte schon die Operation darauf abzielen, sofort voll belastbare Resultate zu erzielen. Bei unbedingt nötiger Teilbelastung sind entsprechende Hilfsmittel zu nutzen, wie z.B. spezielle Gehstützen für Rheumapatienten mit Auflagefläche für den kompletten Unterarm, da durch Problematiken der Gelenke der oberen Extremität normale Gehstützen oft nicht nutzbar sind. Im Extremfall muss bis zur Möglichkeit der Vollbelastung ein Rollstuhl genutzt werden.

Benötigte Orthesen sollten schon präoperativ angepasst sein (s.o.) und im optimalen Fall sollte auch der Gebrauch der Hilfsmittel bereits geübt sein.

Die Physiotherapie beginnt frühestmöglich, sicher aber am ersten postoperativen Tag. Rheumapatienten sind auch Schmerzpatienten und haben durch die lange Krankheitsdauer oft genug auch psychische Probleme. Die Hinzuziehung eines Psychologen kann in diesen Fällen sehr nützlich sein. Die Physiotherapie kann daher manchmal auch nicht wie bei anderen gesunden Patienten durchgeführt werden. Bei den Rheumapatienten ist das Anforderungsniveau oft deutlich zu senken. Bei den Übungen ist auf den Zustand der angrenzenden Gelenke Rücksicht zu nehmen. Fortschritte lassen sich eventuell nur langsamer erzielen.

\section{Komplikationen \\ $\nabla$}

Besonders zu erwähnen, ist die Gefahr von Wundheilungsstörungen und Infektionen. Eine Kortisontherapie erhöht generell und Dosis abhängig diese Gefahren [23]. Verletzungen der durch jahrelange Therapie geschädigten Haut erhöhen weiter das Risiko. Im Vergleich mit Arthrosepatienten ist das Risiko deutlich erhöht [11]. Dies gilt nicht nur für große Eingriffe (z.B. Endoprothetik der großen Gelenke), sondern auch für kleinere handund fußchirurgische Eingriffe. Eine niedrig dosierte Therapie kann fortgeführt werden [24]. Eine britische Kohortenstudie von Dixon et al. [25] zeigte jedoch auch bei niedriger Dosierung schon eine Erhöhung des Risikos.

Kortison und Biologika können das Thromboembolierisiko erhöhen. Aus diesem Grunde ist die frühe Mobilisation der Patienten besonders wichtig. Hierbei sind Eingrenzungen durch den Operateur aufgrund von speziellen Gegebenheiten zu berücksichtigen.

Die Mobilisation muss auch über den stationären Aufenthalt hinaus gesichert werden, um dauerhaft gute Ergebnisse $\mathrm{zu}$ 
erlangen. Es ist eine intensive Zusammenarbeit mit den nach Entlassung aus dem Krankenhaus die Behandlung fortführenden Hausärzten, internistischen Rheumatologen, Physiotherapeuten und Orthopädietechnikern dringend erforderlich.

\section{Interessenkonflikt: es bestehen keine Interessenskonflikte}

\section{Literatur}

1 Rehart S, Henniger $M$. Rolle des Orthopäden bei der Therapie rheumatischer Erkrankungen mit Biologika. Der Orthopäde 2014; 43: 585 $-595$

2 Grennan DM, Gray J, Loudon J et al. Methotrexate and early postoperative complications in patients with rheumatoid arthritis undergoing elective orthopaedic surgery. Ann Rheum Dis 2001; 60: 214-217

3 Sany J, Anaya JM, Canovas F et al. Influence of methotrexate on the frequency of postoperative infectious complications in patients with rheumatoid arthritis. J Rheumatol 1993; 20: 1129-1132

4 Krüger K, Albrecht K, Rehart $S$ et al. Empfehlungen der Deutschen Gesellschaft für Rheumatologie zur perioperativen Vorgehensweise unter Therapie mit DMARDs und Biologika bei entzündlich-rheumatischen Erkrankungen. http://dgrh.de/fileadmin/media/Praxis Klinik/Therapie-Empfehlungen/perioperativ/periop_final230813_ ueberarbeitung_kk.pdf

5 Visser K, Katchamart W, Loza E et al. Multinational evidence-based recommendations for the use of methotrexate in rheumatic disorders with a focus on rheumatoid arthritis: integrating systematic literature research and expert opinion of a broad international panel of rheumatologists in the 3E Initiative. Ann Rheum Dis 2009; 68: 1086-1093

6 Tanaka N, Sakahashi H, Sato E et al. Examination of the risk of continuous leflunomide treatment on the incidence of infectious complications after joint arthroplasty in patients with rheumatoid arthritis. J Clin Rheumatol 2003; 9: 115-118

7 Fuerst $M$, Mohl $H$, Baumgartel $K$ et al. Leflunomide increases the risk of early healing complications in patients with rheumatoid arthritis undergoing elective orthopedic surgery. Rheumatol Int 2006; 26: $1138-1142$

8 Barnard AR, Regan M, Burke FD et al. Wound healing with medications for rheumatoid arthritis in hand surgery. ISRN Rheumatol 2012; 2012: 251962

9 Escalante A, Beardmore TD. Risk factors for early wound complications after orthopedic surgery for rheumatoid arthritis. J Rheumatol 1995; 22: 1844-1851

10 Den Broeder AA, Creemers MC, Fransen J et al. Risk factors for surgical site infections and other complications in elective surgery in patients with rheumatoid arthritis with special attention for anti-tumor necrosis factor: a large retrospective study. J Rheumatol 2007; 34: 689-695

11 Bongartz T, Halligan CS, Osmon DR et al. Incidence and risk factors of prosthetic joint infection after total hip or knee replacement in patients with rheumatoid arthritis. Arthritis Rheum 2008; 59: $1713-1720$
12 Kawakami K, Ikari K, Kawamura K et al. Complications and features after joint surgery in rheumatoid arthritis patients treated with tumour necrosis factor-alpha blockers: perioperative interruption of tumour necrosis factor-alpha blockers decreases complications? Rheumatology (Oxford) 2010; 49: 341-347

13 Gilson M, Gossec L, Mariette X et al. Risk factors for total joint arthroplasty infection in patients receiving tumor necrosis factor $\alpha$-blockers: a case-control study. Arthritis Res Ther 2010; 12: R145

14 Hirao M, Hashimoto J, Tsuboi $\mathrm{H}$ et al. Laboratory and febrile features after joint surgery in patients with rheumatoid arthritis treated with tocilizumab. Ann Rheum Dis 2009; 68: 654-657

15 Toussirot E, Pertuiset E, Sordet $C$ et al. Safety of rituximab in rheumatoid arthritis patients with a history of severe or recurrent bacterial infection: observational study of 30 cases in everyday practice. Joint Bone Spine 2010; 77: 142-145

16 Nishida K, Nasu Y, Hashizume $K$ et al. Abataceptmanagement during the perioperative period in patients with rheumatoid arthritis: report on eight orthopedic procedures. Mod Rheumatol 2014; 24: $544-545$

17 Winking $M$. Die rheumatische Wirbelsäule; Journal für die Neurologie. Neurochirurgie und Psychiatrie 2014; 15: 82-88

18 S3 Leitlinie Vermeidung von perioperativer Hypothermie 2014; Version 8 vom 30.04.2014, Deutsche Gesellschaft für Anästhesiologie und Intensivmedizin, Deutsche Gesellschaft für Chirurgie, Österreichische Gesellschaft für Anästhesie, Reanimation und Intesnivmedizion et al. http:// www.awmf.org/uploads/tx_szleitlinien/001-0181_S3_Vermeidung_ perioperativer_Hypothermie_2014-05.pdf

19 Romlin B, Petruson K, Nilsson K. Moderate superficial hypothermia prolongs bleeding time in humans. Acta Anaesthesiol Scand 2007; 51: 198-201

20 Rajagopalan S, Mascha E, Na J et al. The effects of mild perioperative hypothermia on blood loss and transfusion requirement. Anesthesiology 2008; 108: 71-77

21 Frank SM, Fleisher LA, Breslow AB et al. Perioperative maintenance of normothermia reduces the incidence of morbid cardiac events: A randomized clinical trial. JAMA 1997; 277: 1127-1134

22 Forbes SS, Eskicioglu C, Nathens AB et al. Evidence-based guidelines for prevention of perioperative hypothermia. J Am Coll Surg 2009; 209: 492-503

23 Wolfe F, Caplan L, Michaud K. Treatment for rheumatoid arthritis and the risk of hospitalization for pneumonia: associations with prednisone, disease-modifying antirheumatic drugs, and anti-tumor necrosis factor therapy. Arthritis Rheum 2006; 54: 628-634

24 Jain $A$, Witbreuk $M$, Ball $C$ et al. Influence of steroids and methotrexate on wound complications after elective rheumatoid hand and wrist surgery. J Hand Surg Am 2002; 27: 449-455

25 Dixon WG, Kezouh A, Bernatsky S et al. The influence of systemic glucocorticoid therapy upon the risk of non-serious infection in older patients with rheumatoid arthritis: a nested case-control study. Ann Rheum Dis 2011; 70: 956-960 